LA-14242

Approved for public release;

distribution is unlimited.

\title{
Construction of Superconvergent Discretizations with Differential-Difference Invariants
}

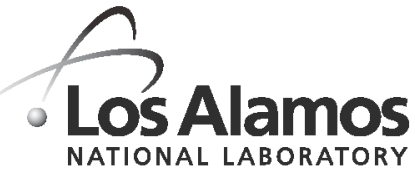

The World's Greatest Science Protecting America 
Edited by Louise Mendius, Group IM-1/X-DO

Photocomposition by Wendy Burditt, Group IM-1

This work was supported in part by the U.S. Department of Energy.

Los Alamos National Laboratory, an affirmative action/equal opportunity employer, is operated by the University of California for the United States Department of Energy under contract W-7405-ENG-36.

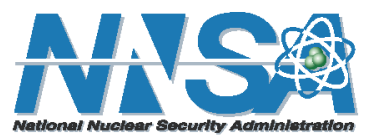

This report was prepared as an account of work sponsored by an agency of the United States Government. Neither the Regents of the University of California, the United States Government nor any agency thereof, nor any of their employees make any warranty, express or implied, or assume any legal liability or responsibility for the accuracy, completeness, or usefulness of any information, apparatus, product, or process disclosed, or represent that its use would not infringe privately owned rights. Reference herein to any specific commercial product, process, or service by trade name, trademark, manufacturer, or otherwise does not necessarily constitute or imply its endorsement, recommendation, or favoring by the Regents of the University of California, the United States Government, or any agency thereof. The views and opinions of authors expressed herein do not necessarily state or reflect those of the Regents of the University of California, the United States Government, or any agency thereof. Los Alamos National Laboratory strongly supports academic freedom and a researcher's right to publish; as an institution, however, the Laboratory does not endorse the viewpoint of a publication or guarantee its technical correctness. 
LA-14242

Issued: August 2005

Construction of Superconvergent Discretizations with Differential-Difference Invariants

Roy A. Axford*

*Consultant at Los Alamos, Department of Nuclear, Plasma, and Radiological Engineering, University of Illinois at Urbana-Champaign, 103 S. Goodwin, Urbana, Illinois 61801 


\section{CONTENTS}

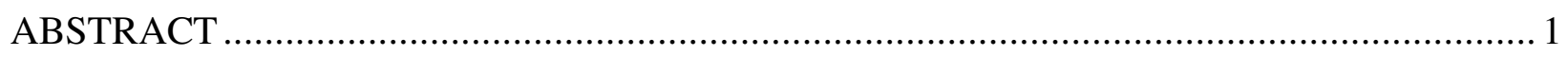

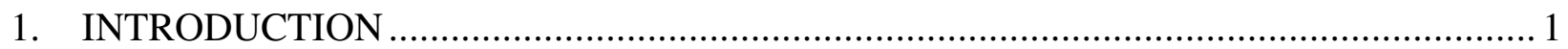

2. CONSTRUCTION OF DIFFERENTIAL-DIFFERENCE INVARIANTS ....................... 2

3. GROUP INVARIANT DISCRETIZATIONS FOR TWO-POINT EIGENVALUE

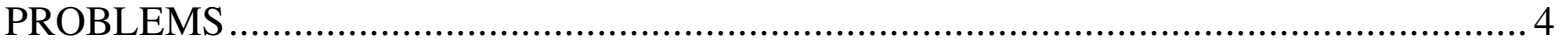

3.1 Energy Eigenvalues for a Particle in a Square Well ............................................... 5

3.2 Material Buckling Eigenvalue Spectra in the Diffusion Approximation.................... 11

4. GROUP INVARIANT DISCRETIZATIONS FOR TWO-POINT INHOMOGENEOUS

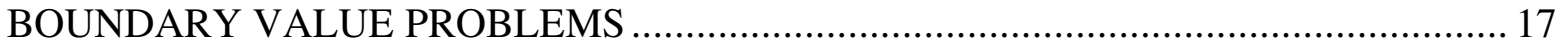

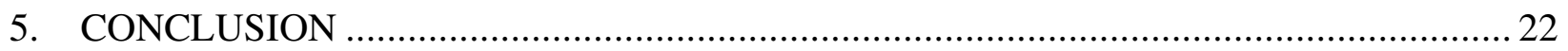

\section{LIST OF TABLES}

1. Even and Odd Parity State Energies for a Square Well with Well Depth $W=16 \ldots \ldots \ldots \ldots \ldots . . . . .10$

2. Exact Solution Material Buckling Eigenvalues for $\frac{a}{T}=6, a_{R} T=3$, and $D_{3}=1 \cdot 2 D_{2} \ldots \ldots \ldots 13$

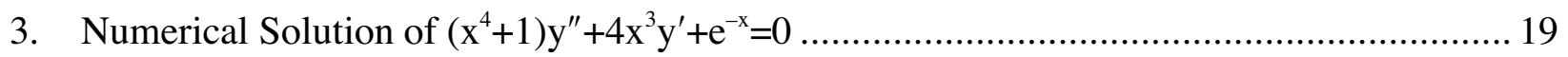

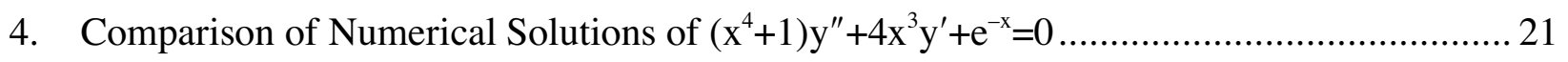




\title{
Construction of Superconvergent Discretizations with Differential-Difference Invariants
}

\author{
by
}

\author{
Roy A. Axford*
}

\begin{abstract}
To incorporate symmetry properties of second-order differential equations into finite difference equations, the concept of differential-difference invariants is introduced. This concept is applied to discretizing homogeneous eigenvalue problems and inhomogeneous two-point boundary value problems with various combinations of Dirichlet, Neumann, and Robin boundary conditions. It is demonstrated that discretizations constructed with differential-difference invariants yield exact results for eigenvalue spectra and superconvergent results for numerical solutions of differential equations.
\end{abstract}

\section{INTRODUCTION}

This study introduces the concept of differential-difference invariants that is applied to the construction of group invariant discretizations of Sturm-Liouville equations. A differentialdifference invariant is defined to be a linear combination of a derivative and the grid-point values of a discretized dependent variable. This combination is constructed so that it admits a subgroup of the group of transformations that is admitted by a differential equation being discretized to obtain numerical solutions.

Finite difference equations that are constructed on the basis of the concept of differentialdifference invariants exhibit the property of being exact. This property means that the exact solutions of the difference equations yield the exact solutions of the differential equations at the grid points. Exact finite difference equations yield exact grid-point values for any arbitrarily selected grid-point spacing. This fact leads to a simplified algorithm for solving the system of algebraic equations that is produced in the discretization process.

Approximation methods, such as the Rayleigh-Ritz and Galerkin techniques, sometime yield the exact solutions of differential equations. When this fact occurs, the results are said to be superconvergent. Finite difference discretizations constructed with the concept of differentialdifference invariants for the two-point boundary value problems have been found to produce superconvergent results. This fact appears to be a consequence of building a symmetry property

* Consultant at Los Alamos: Department of Nuclear, Plasma, and Radiological Enginering, University of Illinois at Urbana-Champaign, 103 S. Goodwin, Urbana, Illinois 61801. 
of the differential equation, and thereby its solution into the discretization method from the beginning.

\section{CONSTRUCTION OF DIFFERENTIAL-DIFFERENCE INVARIANTS}

First and second order differential-difference invariants are needed to construct invariant discretizations used to obtain numerical solutions of two-point boundary value problems. These problems involve homogeneous and inhomogeneous second order differential equations with various combinations of Dirichlet, Neumann, and Robin (or mixed) boundary conditions at the two end points. Let

$$
\hat{U}=\eta(x) \frac{\partial}{\partial y(x)}
$$

be the symbol of the infinitesimal transformation of a group of point transformations admitted by a second order differential equation,

$$
\hat{L} y(x)+S(x)=0
$$

where $\hat{L}$ is a linear second order differential operator. The extension of this group to first order derivatives, second order derivatives, and grid-point values of the dependent variable has the infinitesimal transformation with the symbol,

$$
\begin{aligned}
& \hat{U}^{(G)}=\eta(x) \frac{\partial}{\partial y(x)}+\eta^{\prime}(x) \frac{\partial}{\partial y^{\prime}(x)}+\eta^{\prime \prime}(x) \frac{\partial}{\partial y^{\prime \prime}(x)} \\
& +\eta(x+h) \frac{\partial}{\partial y(x+h)}+\eta(x-h) \frac{\partial}{\partial y(x-h)}+\eta(x+2 h) \frac{\partial}{\partial y(x+2 h)} \\
& +\eta(x-2 h) \frac{\partial}{\partial y(x-2 h)}+\cdots \cdots
\end{aligned}
$$

A first order differential-difference invariant is a linear function of the first order derivative and grid-point values such that

$$
\hat{U}^{(G)} f\left[y(x), y^{\prime}(1), y(x+h), y(x-h), \ldots\right]=0
$$

A second order differential-difference invariant is a linear function of the second order derivative and grid-point values such that

$$
\hat{U}^{(G)} F\left[y(x), y^{\prime \prime}(x), y(x+h), y(x-h), y(x+2 h), y(x-2 h), \ldots\right]=0
$$

The forms of the functions $f$ and $F$ above for the first and second order differential-difference invariants are not unique because their fine-grid limits are used to motivate the linear 
combinations of the grid-point values taken. Fine-grid limits of differential-difference invariants can correspond to central difference approximations, forward difference approximations, or backward difference approximations of first and second order derivatives. However, finite difference equations constructed with differential-differential invariants have been found to be superconvergent, that is, capable of producing exact solutions. This ability is in contrast to finite difference equations constructed with standard difference approximations for derivatives that are not superconvergent.

Five first order differential-difference invariants constructed with the group generator

$$
\hat{U}=\eta(x) \frac{\partial}{\partial y(x)}
$$

are as follows:

$$
\begin{gathered}
y^{\prime}(x)=\left(\frac{\eta^{\prime}(x)}{\eta(x+h)-\eta(x-h)}\right)[y(x+h)-y(x-h)], \\
y^{\prime}(x)=\left(\frac{\eta^{\prime}(x)}{\eta(x+h)-\eta(x)}\right)[y(x+h)-y(x)], \\
y^{\prime}(x)=\left(\frac{\eta^{\prime}(x)}{\eta(x)-\eta(x-h)}\right)[y(x)-y(x-h)], \\
y^{\prime}(x)=\left(\frac{\eta^{\prime}(x)}{\eta(x+2 h)+\eta(x+h)-2 \eta(x)}\right)[y(x+2 h)+y(x+h)-2 y(x)],
\end{gathered}
$$

and

$$
y^{\prime}(x)=\left(\frac{\eta^{\prime}(x)}{2 \eta(x)-\eta(x-h)-\eta(x-2 h)}\right)[2 y(x)-y(x-h)-y(x-2 h)] .
$$

The fine-grid spacings of these five first order differential-difference invariants yield, respectively, the following approximations for first order derivatives:

(1) two-point central difference,

(2) two-point forward difference,

(3) two-point backward difference,

(4) three-point forward difference, and

(5) three-point backward difference. 
Three second order differential-difference invariants constructed with the above group generator are as follows:

$$
\begin{gathered}
y^{\prime \prime}(x)=\left(\frac{\eta^{\prime \prime}(x)}{\eta(x+h)+\eta(x-h)-2 \eta(x)}\right)[y(x+h)+y(x-h)-2 y(x)], \\
y^{\prime \prime}(x)=\left(\frac{\eta^{\prime \prime}(x)}{\eta(x+2 h)-2 \eta(x+h)+\eta(x)}\right)[y(x+2 h)-2 y(x+h)+y(x)],
\end{gathered}
$$

and

$$
\begin{array}{r}
y^{\prime \prime}(x)=\left(\frac{\eta^{\prime \prime}(x)}{\eta(x+h)+\eta(x-h)+\eta(x+2 h)+\eta(x-2 h)}\right) \\
{[y(x+h)+y(x-h)+y(x+2 h)+y(x-2 h)] .}
\end{array}
$$

The fine-grid spacings of these three second order differential-difference invariants yield the following approximations for second order derivatives:

(1) three-point central difference,

(2) three-point forward difference, and

(3) four-point central difference.

The fine-grid spacing limits for the first and second order differential-difference invariants given above all follow directly from the Taylor series expansions of the group generator coordinate function $\eta(x)$ evaluated at the various grid points.

Even though differential-difference invariants are constructed so as to have anticipated fine-grid spacing limits, the group invariant discretizations that are obtained with the concept of differential-difference invariants provide exact solutions of differential equations at grid points set up for any arbitrarily chosen grid spacing. Because second order differential equations are invariant under multi-parameter groups of transformations, different subgroups can be used to construct a differential-difference invariant, which is an additional reason differential-difference invariants are not unique for a given differential equation. The choice of a subgroup can be motivated by the boundary conditions to be satisfied by the differential equation.

\section{GROUP INVARIANT DISCRETIZATIONS FOR TWO-POINT EIGENVALUE PROBLEMS}

Finite difference equations constructed by applying the differential-difference invariant concept to two-point eigenvalue problems involving the scalar Helmholtz operator and Dirichlet, Neumann, and Robin (mixed) boundary conditions have been found to yield exact results for the eigenvalue spectra. Examples drawn from quantum mechanics and neutronic criticality analysis are given in this section. 


\subsection{Energy Eigenvalues for a Particle in a Square Well}

The potential energy for a particle in a square well is given by

$$
U(x)=\left\{\begin{array}{c}
U_{0},-\infty<x<-\frac{L}{2}, \\
0,-\frac{L}{2}<x<\frac{L}{2}, \\
U_{0}, \frac{L}{2}<x<\infty
\end{array}\right.
$$

for a well with width equal to $L$. Because this potential energy is an even function, energy eigenstates with both even and odd parity exist, and it is necessary to consider only the interval $0 \leq x<\infty$. The time-independent Schroedinger equation is

$$
\psi_{1}^{\prime \prime}(x)+\gamma^{2} \psi_{1}(x)=0, \text { for } 0 \leq x \leq \frac{L}{2}
$$

and

$$
\psi_{2}^{\prime \prime}(x)-\alpha^{2} \psi_{2}(x)=0, \text { for } \frac{L}{2} \leq x \leq \infty
$$

where

$$
\gamma^{2}=2 m E / \hbar^{2}
$$

and

$$
\alpha^{2}=2 m\left(U_{0}-E\right) / \hbar^{2},
$$

with $m$ the particle mass and $E$, the energy. The wave function and its derivative are both continuous at $x=L / 2$.

Let $E_{L}$ be the ground state energy of a particle in a box with width $L$. This energy is given by

$$
E_{L}=\hbar^{2} \pi^{2} / 2 m L^{2}
$$

If energy for the particle in the square well is measured in units of $E_{L}$, then

$$
\gamma^{2}=\frac{\pi^{2} E}{L^{2} E_{L}},
$$

and

$$
\alpha^{2}=\frac{\pi^{2}}{L^{2}}\left(\frac{U_{0}}{E_{L}}-\frac{E}{E_{L}}\right) \text {. }
$$

For even parity solutions the analytic solutions of the Schroedinger equation are 


$$
\psi_{1}(x)=A_{1} \cos (\gamma x), 0 \leq x \leq \frac{L}{2}
$$

and

$$
\psi_{2}(x)=A_{2} e^{-\alpha x} \quad, \frac{L}{2} \leq x \leq \infty
$$

For odd parity solutions the analytic solutions are

$$
\psi_{1}(x)=A_{3} \sin (\gamma x), 0 \leq x \leq \frac{L}{2},
$$

and

$$
\psi_{2}(x)=A_{4} e^{-\alpha x} \quad, \quad \frac{L}{2} \leq x \leq \infty
$$

In both cases a transcendental equation is obtained for the allowed energies by making the wave function and its derivative continuous at $x=L / 2$. The allowed energies for the even parity states are roots of the transcendental equation,

$$
\sqrt{\frac{E}{E_{L}}} \tan \left(\frac{\pi}{2} \sqrt{\frac{E}{E_{L}}}\right)=\sqrt{\frac{U_{0}}{E_{L}}-\frac{E}{E_{L}}} .
$$

The allowed energies for the odd parity states are roots of

$$
\sqrt{\frac{E}{E_{L}}} \cot \left(\frac{\pi}{2} \sqrt{\frac{E}{E_{L}}}\right)=-\sqrt{\frac{U_{0}}{E_{L}}-\frac{E}{E_{L}}} .
$$

With the definitions,

and

$$
X^{2}=\frac{E}{E_{L}}
$$

$$
W=\frac{U_{0}}{E_{L}}
$$

these transcendental equations can be written as

$$
X \sin (\pi X / 2)-\cos (\pi X / 2) \sqrt{W-X^{2}}=0
$$

for even parity states, and for odd parity states

$$
X \cos (\pi X / 2)+\sin (\pi X / 2) \sqrt{W-X^{2}}=0
$$


When $W=16=U_{0} / E_{L}$, there are five allowed energies for three even parity states and two odd parity states. The roots of these transcendental equations that come out of the exact analytic solutions of the Schroedinger equation are given in the third column of Table I.

It will now be shown that this eigenvalue spectrum can be obtained from finite difference equations constructed by applying the differential-difference invariant concept. In the discretized method of solution we solve the Schroedinger equation

$$
\psi_{1}^{\prime \prime}(x)+\gamma^{2} \psi_{1}(x)=0,0 \leq x \leq \frac{L}{2}
$$

subject to the Robin boundary condition

$$
\alpha \psi_{1}\left(\frac{L}{2}\right)+\psi_{1}^{\prime}\left(\frac{L}{2}\right)=0
$$

which comes out of the continuity of the wave function and its derivative at $x=L / 2$. To discretize the differential equation, we use either the group with the generator

$$
\hat{U}=\cos (\gamma x) \frac{\partial}{\partial y}
$$

for even parity states, or the group with the generator

$$
\hat{U}=\sin (\gamma x) \frac{\partial}{\partial y}
$$

for odd parity states. In both cases a second order differential-difference invariant is found to be given by

$$
\psi_{1}^{\prime \prime}(x)=\frac{\gamma^{2}}{4 \sin ^{2}\left(\frac{\gamma h}{2}\right)}\left[\psi_{1}(x+h)+\psi_{1}(x-h)-2 \psi_{1}(x)\right]
$$

Hence, the discretized form of the Schroedinger equations for both even and odd parity state solutions is

$$
\psi_{1}(x+h)+\psi_{1}(x-h)-\left[2-4 \sin ^{2}\left(\frac{\gamma h}{2}\right)\right] \psi_{1}(x)=0
$$

for a grid spacing equal to $h$. 
The discretization of the Robin boundary condition at $x=L / 2$ is different for even parity and odd parity eigenstates. For even parity states a first order differential-difference invariant to use in the Robin boundary condition is given by

$$
\psi_{1}^{\prime}(x)=\left(\frac{\gamma \sin (\gamma x)}{\cos [\gamma(x-h)]-\cos (\gamma x)}\right)\left[\psi_{1}(x)-\psi_{1}(x-h)\right]
$$

or

$$
\psi_{1}^{\prime}(x)=\left(\frac{\gamma \sin (\gamma x)\left[\psi_{1}(x)-\psi_{1}(x-h)\right]}{2 \sin \left[\gamma\left(x-\frac{h}{2}\right)\right] \sin \left(\gamma \frac{h}{2}\right)}\right) .
$$

For odd parity states a first order differential-difference invariant is given by

$$
\psi_{1}^{\prime}(x)=\frac{\gamma \cos (\gamma x)\left[\psi_{1}(x)-\psi_{1}(x-h)\right]}{2 \cos \left[\gamma\left(x-\frac{h}{2}\right)\right] \sin \left(\frac{\gamma h}{2}\right)}
$$

Hence, the Robin boundary condition at $x=L / 2$, namely,

$$
\alpha \psi_{1}\left(\frac{L}{2}\right)+\psi_{1}^{\prime}\left(\frac{L}{2}\right)=0
$$

takes one of the two following forms. For even parity states this boundary condition is

$$
-\gamma \sin \left(\frac{\gamma L}{2}\right) \psi_{1}\left(\frac{L}{2}-h\right)+\psi_{1}\left(\frac{L}{2}\right)\left\{2 \alpha \sin \left[\gamma\left(\frac{L}{2}-\frac{h}{2}\right)\right] \sin \left(\frac{\gamma h}{2}\right)+\gamma \sin \left(\frac{\gamma h}{2}\right)\right\}=0
$$

For odd parity states the Robin boundary condition is

$$
-\gamma \cos \left(\frac{\gamma L}{2}\right) \psi_{1}\left(\frac{L}{2}-h\right)+\psi_{1}\left(\frac{L}{2}\right)\left\{2 \alpha \cos \left[\gamma\left(\frac{L}{2}-\frac{h}{2}\right)\right] \sin \left(\frac{\gamma h}{2}\right)+\gamma \cos \left(\frac{\gamma L}{2}\right)\right\}=0
$$

To determine the allowed energies for the even and odd parity eigenstates from the difference equations obtained in the discretized method of solution, we set up systems of homogeneous algebraic equations from the discretized forms of the Schroedinger equation and the Robin boundary condition. A system of homogeneous algebraic equations has a nontrivial solution, provided that the determinant of the coefficient matrix vanishes. This requirement yields the eigenvalue spectrum for the allowed energies. For even parity eigenstates we set up a system of three algebraic equations, and for the odd parity eigenstates, two algebraic equations. 
In the case of the even parity eigenstates we can take the wave function evaluated a $x=0$, $x=L / 4$, and $x=L / 2$ as three unknowns. The corresponding grid spacing is $h=L / 4$. We evaluate the discretized Schroedinger equation at $x=0$ to obtain

$$
2 \psi_{1}\left(\frac{L}{4}\right)-\left[2-4 \sin ^{2}\left(\frac{\gamma L}{8}\right)\right] \psi_{1}(0)=0
$$

and at $x=L / 4$ to obtain

$$
\psi_{1}\left(\frac{L}{2}\right)+\psi_{1}(0)-\left[2-4 \sin ^{2}\left(\frac{\gamma L}{8}\right)\right] \psi_{1}\left(\frac{L}{4}\right)=0
$$

Evaluating the Robin boundary condition at $x=L / 2$ for even parity states yields

$$
-\gamma \sin \left(\frac{\gamma L}{2}\right) \psi_{1}\left(\frac{L}{4}\right)+\psi_{1}\left(\frac{L}{2}\right)\left[2 \alpha \sin \left(\frac{3 \gamma L}{8}\right) \sin \left(\frac{\gamma L}{8}\right)+\gamma \sin \left(\frac{\gamma L}{2}\right)\right]=0
$$

The determinant of the coefficient matrix for these last three algebraic equations is

where

$$
\left|\begin{array}{ccc}
4 \sin ^{2}\left(\frac{\gamma L}{8}\right)-2 & 2 & 0 \\
1 & 4 \sin ^{2}\left(\frac{\gamma L}{8}\right)-2 & 1 \\
0 & -(\gamma L) \sin \left(\frac{\gamma L}{2}\right) & 2(\alpha L) \sin \left(\frac{3 \gamma L}{8}\right) \sin \left(\frac{\gamma L}{8}\right)+(\gamma L) \sin \left(\frac{\gamma L}{2}\right)
\end{array}\right|
$$

$$
\begin{gathered}
\gamma L=\pi X, \\
\alpha L=\pi \sqrt{W-X^{2}}, \\
X^{2}=\frac{E}{E_{L}},
\end{gathered}
$$

and

$$
W=\frac{U_{0}}{E_{L}}
$$

For a specified value of the well depth $W$, the values of $X$ that make the above determinant equal to zero give the allowed energies for the even parity eigenstates.

For the odd parity states we take the values of the wave function at $x=L / 4$ and $x=L / 2$ as two unknowns, since $\psi_{1}(0)=0$ in the odd parity case. The discretized form of the Schroedinger equation with $x=L / 4$ and $h=L / 4$ gives 


$$
\psi_{1}\left(\frac{L}{2}\right)-\left[2-4 \sin ^{2}\left(\frac{\gamma L}{8}\right)\right] \psi_{1}\left(\frac{L}{4}\right)=0
$$

The discretized form of the Robin boundary condition for the odd parity case becomes

$$
-(\gamma L) \cos \left(\frac{\gamma L}{2}\right) \psi_{1}\left(\frac{L}{4}\right)+\psi_{1}\left(\frac{L}{2}\right)\left[2(\alpha L) \cos \left(\frac{3 \gamma L}{8}\right) \sin \left(\frac{\gamma L}{8}\right)+(\gamma L) \cos \left(\frac{\gamma L}{2}\right)\right]=0
$$

The determinant of the coefficient matrix for these two algebraic equations is given by

$$
\left|\begin{array}{cc}
4 \sin ^{2}\left(\frac{\gamma L}{8}\right)-2 & 1 \\
-(\gamma L) \cos \left(\frac{\gamma L}{2}\right) & 2(\alpha L) \cos \left(\frac{3 \gamma L}{8}\right) \sin \left(\frac{\gamma L}{8}\right)+(\gamma L) \cos \left(\frac{\gamma L}{2}\right)
\end{array}\right|
$$

For a specified value of the well depth $W$, the values of $X$ that make this determinant vanish yield the allowed values for the energies of the odd parity eigenstates.

When $W=16$, there are five allowed energy levels, three with even parity and two with odd parity. For this value of $W$, the allowed energies for the five eigenstates computed with the above two determinants are given in the fourth column of Table 1. The allowed energies computed as the roots of the two transcendental equations obtained with the exact solutions of the Schroedinger equation for even and odd parity states as computed from Eqs. (31) and (32), respectively, are given in the third column of Table 1. Comparison of the third and fourth columns of Table 1 shows that the discretization of the Schoedinger equation and the Robin boundary condition constructed with the concept of differential-difference invariants produces

\begin{tabular}{|c|c|c|c|}
\hline \multicolumn{4}{|c|}{$\begin{array}{l}\text { TABLE 1. Even and Odd Parity State Energies for a Square Well with } \\
\text { Well Depth } W=16 \text {. }\end{array}$} \\
\hline State & Parity of State & $\begin{array}{l}\text { Exact Solution } \\
\text { State Energy } X\end{array}$ & $\begin{array}{l}\text { Discretized Solution } \\
\text { State Energy } X\end{array}$ \\
\hline 1 & Even & 0.861762 & 0.861762 \\
\hline 2 & Odd & 1.71747 & 1.71747 \\
\hline 3 & Even & 2.55823 & 2.55823 \\
\hline 4 & Odd & 3.36395 & 3.36395 \\
\hline 5 & Even & 4.0 & 4.0 \\
\hline
\end{tabular}
exact results for the allowed energies or eigenvalue spectrum. Numerical results computed with the discretization method for $W=3$ and $W=10$ are also exact values of the allowed energies. 


\subsection{Material Buckling Eigenvalue Spectra in the Diffusion Approximation}

For a two-region core with material buckling distribution given by

$$
B_{m}^{2}=\left\{\begin{array}{cl}
0, & \text { for } 0 \leq x \leq b, \\
B^{2}, & \text { for } \quad b \leq x \leq a
\end{array}\right.
$$

and for a reflector with thickness $T$ and inverse diffusion length $\alpha_{R}$, the three region neutron diffusion equations are

$$
\begin{gathered}
\psi_{1}^{\prime \prime}(x)=0, \text { for } 0 \leq x \leq b, \\
\psi_{2}^{\prime \prime}(x)+B^{2} \psi_{2}(x)=0, \text { for } b \leq x \leq a,
\end{gathered}
$$

and

$$
\psi_{3}^{\prime \prime}(x)-\alpha_{R}^{2} \psi_{3}(x)=0, \text { for } a \leq x \leq a+T .
$$

In one-group modeling, a vanishing material buckling in the center of the core produces a central region in which the fission rate is spatially uniform so the power density is a constant. The nonzero material buckling in the outer zone of the core has an eigenvalue spectrum where the fundamental mode corresponds to the just critical state. The higher modes are needed for transient calculations.

The eigenvalue problem for the material buckling in the outer region of the core is composed of the solution of the differential equation for the flux $\psi_{2}(x)$ for $b \leq x \leq a$, subject to the Nuemann boundary condition at $x=b$, namely

$$
\psi_{2}^{\prime}(b)=\psi_{1}^{\prime}(b)=0
$$

where $\psi_{1}(x)=G=$ constant, and to the Robin boundary condition at $x=a$, namely

$$
(B a) \sin (B a)-R \cos (B a)=0,
$$

where $b=0$, and where

$$
R=\frac{D_{3}}{D_{2}}\left(\frac{a}{T}\right)\left(\alpha_{R} T\right) \operatorname{coth}\left(\alpha_{R} T\right),
$$

and the diffusion coefficient is given by

$$
D(x)=\left\{\begin{array}{ll}
D_{2}, & \text { for } \quad b \leq x \leq a \\
D_{3}, & \text { for } \quad a \leq x \leq a+T .
\end{array} .\right.
$$

This Robin boundary condition at $x=a$ is a consequence of the continuity of the neutron flux and net current at $x=a$. When $b \neq 0$, the Robin boundary condition at $x=a$ is given by 


$$
(B a) \sin \left[B a\left(1-\frac{b}{a}\right)\right]-R \cos \left[B a\left(1-\frac{b}{a}\right)\right]=0
$$

The solutions of the diffusion equations (56) and (57) from which the Robin boundary conditions (59) and (62) are derived are

$$
\begin{gathered}
\psi_{2}(x)=C_{2} \cos (B x), \text { if } b=0, \\
\psi_{2}(x)=C_{2} \cos [B(x-b)], \text { if } b \neq 0,
\end{gathered}
$$

and

$$
\psi_{3}(x)=C_{3} \sinh \left[\alpha_{R}(a+T-x)\right]
$$

where $C_{2}$ and $C_{3}$ are constants, and the Dirichlet boundary condition,

$$
\psi_{3}(a+T)=0
$$

is satisfied.

Material buckling eigenvalues computed from the transcendental equations, (59) for $b=0$ and (62) for $b / a=0.60$, are given in the second and third columns of Table 2, respectively, for the parameter values,

$$
\begin{gathered}
\frac{a}{T}=6, \\
\frac{\alpha_{R}}{T}=3,
\end{gathered}
$$

and

$$
D_{3}=(1 \cdot 2) D_{2}
$$

The first six or seven eigenvalues out of an infinite spectrum are given.

The material buckling eigenvalue spectra given in Table 2 were found with the exact solutions of the differential equations. We will now show that the same results for these eigenvalues are obtained by discretizing the differential equation for $\psi_{2}(x)$ and the Robin boundary condition at $x=a$ with the second and first order differential-difference invariants. That is, we construct invariant discretizations of

and

$$
\psi_{2}^{\prime \prime}(x)+B^{2} \psi_{2}(x)=0, \text { for } b \leq x \leq a
$$

$$
\psi_{2}(a)+\frac{D_{2}}{D_{3}} \frac{\sinh \left(\alpha_{R} T\right)}{\alpha_{R} \cosh \left(\alpha_{R} T\right)} \psi_{2}^{1}(a)=0
$$




\begin{tabular}{|c|c|c|}
\hline \multicolumn{3}{|c|}{ TABLE 2. Exact Solution Material Buckling } \\
Eigenvalues for $\frac{\boldsymbol{a}}{\boldsymbol{T}}=\boldsymbol{6}, \boldsymbol{\alpha}_{\boldsymbol{R}} \boldsymbol{T}=\mathbf{3}$, and $\boldsymbol{D}_{\mathbf{3}}=\mathbf{1} \cdot \mathbf{2}_{\boldsymbol{2}} \cdot$ \\
\hline Mode & $\boldsymbol{B} \boldsymbol{a}$ for $\boldsymbol{b}=\mathbf{0}$ & $\boldsymbol{B} \boldsymbol{a}$ for $\boldsymbol{b} / \boldsymbol{a}=\mathbf{0 . 6 0}$ \\
\hline 1 & 1.50173 & 3.52458 \\
\hline 2 & 4.50764 & 10.6415 \\
\hline 3 & 7.52047 & 17.9103 \\
\hline 4 & 10.5434 & 25.3331 \\
\hline 5 & 13.5782 & 32.8749 \\
\hline 6 & 16.6252 & 40.5000 \\
\hline 7 & 19.6838 & \\
\hline
\end{tabular}

To construct the second and first order differential-difference invariants, we use the group generator

$$
\hat{U}=\eta(x) \frac{\partial}{\partial \psi_{2}(x)}
$$

A second order differential difference invariant is given by

$$
\psi_{2}^{\prime \prime}(x)=\left(\frac{\eta^{\prime \prime}(x)}{\eta(x+h)+\eta(x-h)-2 \eta(x)}\right)\left[\psi_{2}(x+h)+\psi_{2}(x-h)-2 \psi_{2}(x)\right],
$$

where

$$
\eta(x)=\cos (B x), \text { for } b=0,
$$

and

$$
\eta(x)=\cos B(x-b), \text { for } b \neq 0 \text {. }
$$

For each of these two cases, $b=0$ and $b \neq 0$, the second order differential-difference invariant in Eq. (73) reduces to

$$
\psi_{2}^{\prime \prime}(x)=\frac{B^{2}}{4 \sin ^{2}\left(\frac{B h}{2}\right)}\left[\psi_{2}(x+h)+\psi_{2}(x-h)-2 \psi_{2}(x)\right] .
$$


Accordingly, the invariant discretization of the diffusion equation (70) is given by

$$
\psi_{2}(x+h)+\psi_{2}(x+h)+\left[4 \sin ^{2}\left(\frac{B h}{2}\right)-2\right] \psi_{2}(x)=0
$$

for both the $b=0$ and $b \neq 0$ cases. A first order differential-difference invariant to discretize the Robin boundary condition of Eq. (71) is given by

$$
\psi_{2}^{\prime}(x)=\left(\frac{\eta^{\prime}(x)}{\eta(x)-\eta(x-h)}\right)\left[\psi_{2}(x)-\psi_{2}(x-h)\right]
$$

where $\eta(x)$ is given by Eq. (74) for $b=0$ and by Eq. (75) for $b \neq 0$. When $b=0$, we find in Eqs. (74) and (78) that the first order differential-difference invariant, when evaluated at $x=a$, becomes

$$
\psi_{2}^{\prime}(a)=\frac{B \sin (B a)\left[\psi_{2}(a)-\psi_{2}(a-h)\right]}{2 \sin \left(\frac{B h}{2}\right) \sin \left(B a-\frac{B h}{2}\right)}
$$

When $b \neq 0$, with Eqs (75) and (78), the first order differential-difference invariant reduces to

$$
\psi_{2}^{\prime}(a)=\frac{B \sin [B(a-b)]\left[\psi_{2}(a)-\psi_{2}(a-h)\right]}{2 \sin \left(\frac{B h}{2}\right) \sin \left[B(a-b)-\frac{B h}{2}\right]}
$$

at $x=a$.

The Robin boundary condition of Eq. (71) can be discretized for the two cases, $b=0$ and $b \neq 0$, with the first order differential-difference invariants found in Eqs. (79) and (80). With $h=a / 2$ and $b=0$, the discretized Robin boundary condition at $x=a$ becomes

$$
\left[2 R \sin \left(\frac{B a}{4}\right) \sin \left(\frac{3 B a}{4}\right)+(B a) \sin (B a)\right] \psi_{2}(a)-(B a) \sin (B a) \psi_{2}(a-h)=0
$$

With $2 h=b-a$ and $b \neq 0$, the discretized Robin boundary condition at $x=a$ reduces to

$$
\begin{array}{r}
\left\{2 R \sin \left[\frac{B}{4}(a-b)\right] \sin \left[\frac{3}{4} B(a-b)\right]+(B a) \sin [B(a-b)]\right\} \psi_{2}(a) \\
-(B a) \sin [B(a-b)] \psi_{2}(a-h)=0 .
\end{array}
$$

In both Eqs. (81) and (82) the parameters $R$, which contain the reflector properties, is given in Eq. (60). 
The material buckling eigenvalue spectra can be computed for the $b=0$ case by setting up three homogeneous algebraic equations with the discretized form of the differential equation in Eq. (77), and the discretized form of the Robin boundary condition in Eq. (81). In the $b \neq 0$ case three homogeneous algebraic equations are set up again with Eq. (77) and the discretized form of the Robin boundary condition found in Eq. (82).

When $b=0$ we take as three unknowns $\psi_{2}(0), \psi_{2}(h)$, and $\psi_{2}(a)$ with $2 h=a$. Evaluating Eq. (77) at $x=0$ yields

$$
2 \psi_{2}(h)+\left[4 \sin ^{2}\left(\frac{B a}{4}\right)-2\right] \psi_{2}(0)=0
$$

Evaluating Eq. (77) at $x=h=a / 2$ yields

$$
\psi_{2}(a)+\psi_{2}(0)+\left[4 \sin ^{2}\left(\frac{B a}{4}\right)-2\right] \psi_{2}(h)=0
$$

The discretized Robin boundary condition in Eq. (81) becomes

$$
\begin{array}{r}
{\left[2 R \sin \left(\frac{B a}{4}\right) \sin \left(\frac{3 B a}{4}\right)+(B a) \sin (B a)\right] \psi_{2}(a)} \\
-(B a) \sin (B a) \psi_{2}(h)=0 .
\end{array}
$$

The three homogeneous algebraic equations (83), (84), and (85) have a nontrivial solution when the determinant of their coefficient matrix vanishes. This determinant is

where

$$
\left|\begin{array}{ccc}
4 \sin ^{2}\left(\frac{B a}{4}\right)-2 & 2 & 0 \\
1 & 4 \sin ^{2}\left(\frac{B a}{4}\right)-2 & 1 \\
0 & -B a \sin (B a) & M_{33}
\end{array}\right|
$$

$$
M_{33}=2 R \sin \left(\frac{B a}{4}\right) \sin \left(\frac{3 B a}{4}\right)+(B a) \sin (B a) .
$$

The zeros of this determinant yield the material buckling eigenvalues for the $b=0$ case. Computing the first seven of these zeros for the parameter values $a / T=b, \alpha_{R} T=3$, and $D_{3}=1.2 D_{2}$, yields the same results given in the second column of Table 2 that were computed with the exact solution of the differential equation. The invariant discretization of the differential equation and Robin boundary condition with the concept of first and second order differential- 
difference invariants is a discretization that produces exact results for the material buckling eigenvalues in the $b=0$ case.

The same is true also for the $b \neq 0$ case. When $b \neq 0$, we take $2 h=b-a$ and derive three homogeneous algebraic equations for the unknowns $\psi_{2}(b), \psi_{2}(b+h)$, and $\psi_{2}(a)$. Evaluating Eq. (77) at $x=b$ gives

$$
2 \psi_{2}(b+h)+\left\{4 \sin ^{2}\left[\frac{B a}{4}\left(1-\frac{b}{a}\right)\right]-2\right\} \psi_{2}(b)=0
$$

Evaluating Eq. (77) at $x=b+h$ gives

$$
\psi_{2}(a)+\psi_{2}(b)+\left\{4 \sin ^{2}\left[\frac{B a}{4}\left(1-\frac{b}{a}\right)\right]-2\right\} \psi_{2}(b+h)=0
$$

The discretized Robin boundary condition in Eq. (82) for the $b \neq 0$ case becomes

$$
\begin{array}{r}
\left\{2 R \sin \left[\frac{B}{4}(a-b)\right] \sin \left[\frac{3 B}{4}(a-b)\right]+(B a) \sin [B(a-b)]\right\} \psi_{2}(a) \\
-(B a) \sin [B(a-b)] \psi_{2}(b+h)=0 .
\end{array}
$$

The determinant of the coefficient matrix of the three homogeneous algebraic equations (86), (87), and (88) is

where

$$
\left|\begin{array}{ccc}
4 \sin ^{2}\left[\frac{B a}{4}\left(1-\frac{b}{a}\right)\right]-2 & 2 & 0 \\
1 & 4 \sin ^{2}\left[\frac{B a}{4}\left(1-\frac{b}{a}\right)\right] & 1 \\
0 & -B a \sin \left[B a\left(1-\frac{b}{a}\right)\right] & N_{33}
\end{array}\right|,
$$

$$
N_{33}=2 R \sin \left[\frac{B a}{4}\left(1-\frac{b}{a}\right)\right] \sin \left[\frac{3 B a}{4}\left(1-\frac{b}{a}\right)\right]+(B a) \sin \left[B a\left(1-\frac{b}{a}\right)\right]
$$

Computing the first six zeros of this determinant with the parameter values $a / T=b, \alpha_{R} T=3$, $D_{3}=1.2 D_{2}$, and $b / a=0.60$ yields the same results for the material buckling eigenvalues that are shown in the third column of Table 2 for the $b \neq 0$ case. 


\section{GROUP INVARIANT DISCRETIZATIONS FOR TWO-POINT INHOMOGENEOUS BOUNDARY VALUE PROBLEMS}

The inhomogeneous second order differential equation

$$
\frac{d}{d x}\left[\left(x^{4}+1\right) \frac{d y}{d x}\right]+e^{-x}=0,
$$

subject to the boundary conditions $y(0)=0$ and $y(1)=0$, has no closed form analytic solution. However, the solution does have an integral representation from which numerical results can be obtained. This differential equation can also be discretized by using the concepts of first and second order differential-difference invariants to obtain numerical result for the solution. These two approaches for obtaining numerical results are compared below and found to yield the same answers.

The integral representation of the solution of Eq. (89) is given by

where

$$
y(x)=\phi_{p}(x)+C \eta(x),
$$

$$
\eta(x)=\int_{0}^{x} \frac{d \xi}{\xi^{4}+1},
$$

and

$$
\phi_{p}(x)=\int_{0}^{x} \frac{e^{-\xi} d \xi}{\xi^{4}+1} .
$$

The constant $C$ in Eq. (90) is given by

$$
C=(-1) \phi_{p}(1) / \eta(1)=-0.659797 .
$$

The alternative form of Eq. (89), with which its discretized form can be constructed, is

$$
\left(x^{4}+1\right) y^{\prime \prime}+4 x^{3} y^{\prime}+e^{-x}=0 \quad .
$$

This equation is invariant under the group of point transformations with the generator

$$
\hat{U}=\eta(x) \frac{\partial}{\partial y},
$$

where the coordinate function $\eta(x)$ is given by Eq. (91). A first order differential-difference invariant of this group is 


$$
y^{\prime}(x)=\frac{\eta^{\prime}(x)[y(x+h)-y(x-h)]}{\eta(x+h)-\eta(x-h)},
$$

and a second order differential-difference invariant is

$$
y^{\prime \prime}(x)=\frac{\eta^{\prime \prime}(x)[y(x+h)+y(x-h)-2 y(x)]}{\eta(x+h)+\eta(x-h)-2 \eta(x)} .
$$

The discretized form of Eq. (94) is

$$
\hat{\Omega} y(x)+Q(x)=0
$$

where the discretized inhomogeneous term is given by

$$
Q(x)=(-1) \hat{\Omega} \phi_{p}(x)
$$

with the particular integral of Eq. (92). The difference operator in Eqs. (98) and (99) is

$$
\hat{\Omega}=\frac{\left(x^{4}+1\right) \eta^{\prime \prime}(x)\left[\hat{S}^{+}+\hat{S}^{-}-2\right]}{\eta(x+h)+\eta(x-h)-2 \eta(x)}+\frac{4 x^{3} \eta^{\prime}(x)\left[\hat{S}^{+}-\hat{S}^{-}\right]}{\eta(x+h)-\eta(x-h)}
$$

or, as

$$
\eta^{\prime}(x)=\frac{1}{x^{4}+1}
$$

and

$$
\eta^{\prime \prime}(x)=\frac{-4 x^{3}}{\left(x^{4}+1\right)^{2}}
$$

we have

$$
\hat{\Omega}=\frac{-4 x^{3}\left[\hat{S}^{+}+\hat{S}^{-}-2\right]}{\left(x^{4}+1\right)[\eta(x+h)+\eta(x-h)-2 \eta(x)]}+\frac{4 x^{3}\left[\hat{S}^{+}+\hat{S}^{-}\right]}{\left(x^{4}+1\right)[\eta(x+h)-\eta(x-h)]} .
$$

In Eqs. (101) and (103) $\hat{S}^{+}$is the shift operator to the right, and $\hat{S}^{-}$is the shift operator to the left. With the Eqs. (103) and (99) the difference equation (98) becomes

$$
\begin{gathered}
\frac{y(x+h)-y(x-h)}{\eta(x+h)-\eta(x-h)}-\frac{y(x+h)+y(x-h)-2 y(x)}{\eta(x+h)+\eta(x-h)-2 y(x)}=\frac{\phi_{p}(x+h)-\phi_{p}(x-h)}{\eta(x+h)+\eta(x-h)} \\
-\frac{\phi_{p}(x+h)+\phi_{p}(x-h)-2 \phi_{p}(x)}{\eta(x+h)+\eta(x-h)-2 \eta(x)} .
\end{gathered}
$$


This can be rearranged to give

$$
\begin{aligned}
& {[\eta(x-h)-\eta(x)] y(x+h)+[\eta(x)-\eta(x+h)] y(x-h)+[\eta(x+h)-\eta(x-h)] y(x)=} \\
& \phi_{p}(x+h)[\eta(x-h)-\eta(x)]+\phi_{p}(x-h)[\eta(x)-\eta(x+h)]+\phi_{p}(x)[\eta(x+h)-\eta(x-h)] .
\end{aligned}
$$

Numerical results for the solution of the differential equation (89) are given in Table 3. Solutions are computed with the integral representation (90) in the second column of Table 3 and the invariant difference equation (105) in the third column. The numerical results computed by these two methods are in exact agreement.

\begin{tabular}{|c|c|c|}
\hline \multicolumn{3}{|c|}{ TABLE 3. Numerical Solution of $\left(\mathbf{x}^{\mathbf{4}}+\mathbf{1}\right) \mathbf{y}^{\prime \prime}+\mathbf{4}^{\mathbf{3}} \mathbf{y}^{\prime}+\mathbf{e}^{-\mathbf{x}}=\mathbf{0 .}$} \\
\hline $\boldsymbol{x}$ & $\begin{array}{c}\text { Integral Representation } \\
\text { Numerical Results }\end{array}$ & $\begin{array}{c}\text { Invariant Difference } \\
\text { Equation Results }\end{array}$ \\
\hline 0 & 0 & 0 \\
\hline $1 / 4$ & 0.056220 & 0.056220 \\
\hline $1 / 2$ & 0.063560 & 0.063560 \\
\hline $3 / 4$ & 0.037537 & 0.037537 \\
\hline 1 & 0 & 0 \\
\hline
\end{tabular}

When the numerical results were computed with the invariant difference equation (105), it was not necessary to write out a system of the algebraic equations to solve simultaneously for $y(1 / 4)$, $y(1 / 2)$, and $y(3 / 4)$. These values can be determined sequentially as follows:

(1) Set $h=1 / 2$ and $x=1 / 2$ in Eq. (105) and solve for $y(1 / 2)$.

(2) Set $h=1 / 4$ and $x=1 / 4$ in Eq. (105) and solve for $y(1 / 4)$ knowing $y(1 / 2)$ from the previous step.

(3) Set $h=1 / 4$ and $x=3 / 4$ in Eq. (105) and solve for $y(3 / 4)$ knowing $y(1 / 2)$.

If values at further grid points are wanted, we can set $h(1 / 8)$, and then calculate $y(1 / 8), y(3 / 8)$, $y(5 / 8)$, and $y(7 / 8)$ sequentially. Repeated halving of the grid spacing $h$ yields additional results at more grid points. A large order algebraic system is not required to compute the solution at a large number of grid points.

An approximate solution of the differential equation (89) can be calculated with the RayleighRitz variational technique. Equation (89) is a variational principle Euler-Lagrange equation of the functional 


$$
J=\int_{0}^{1} d x\left[\left(x^{4}+1\right)\left(y^{\prime}\right)^{2}-2 e^{-x} y\right]
$$

Let

$$
y(x)=e^{-x}\left[a x(1-x)+b x(1-x)^{2}\right]
$$

be a trial function in which the constants $a$ and $b$ are to be determined. The two algebraic equations for these two constants are found from the conditions

and

$$
\frac{d J}{d a}(a, b)=0
$$

$$
\frac{d J}{d b}(a, b)=0
$$

These two algebraic equations are

$$
28\left(7 e^{2}-51\right) a+\left(4025-543 e^{2}\right) b=8,
$$

and

$$
\left(4025-543 e^{2}\right) a+2\left(1119 e^{2}-8263\right) b=2\left(e^{2}-5\right)
$$

The solutions are

$$
a=0.454514
$$

and

$$
b=-0.094657 \text {. }
$$

With these values of the constants, the approximate solution in Eq. (107) gives

and

$$
\begin{aligned}
& y(1 / 4)=0.056004, \\
& y(1 / 2)=0.061743,
\end{aligned}
$$

$$
y(3 / 4)=0.038160
$$

These Rayleigh-Ritz method approximate results are all quite close to the exact results given in Table 3.

For comparison purposes the differential equation (94) has also been solved approximately numerically by constructing a finite difference equation in a conventional way. The difference equation

$$
\left(x^{4}+1+2 h x^{3}\right) y(x+h)+\left(x^{4}+1-2 h x^{3}\right) y(x-h)-2\left(x^{4}+1\right) y(x)+h^{2} e^{-x}=0
$$


with the grid spacing $h$ approximates Eq. (94) when the second order derivative is approximated by

$$
y^{\prime \prime}(x)=\frac{1}{h^{2}}[y(x+h)+y(x-h)-2 y(x)],
$$

and the first order derivative by

$$
y^{\prime}(x)=\frac{1}{2 h}[y(x+h)-y(x-h)] .
$$

These are standard two-point and three-point central difference formulae. When the grid spacing is $h=1 / 8$, Eq. (117) becomes a set of seven algebraic equations for $y(1 / 8), y(1 / 4), y(3 / 8)$, $y(1 / 2), y(5 / 8), y(3 / 4)$, and $y(7 / 8)$. The numerical solution of this set of seven algebraic equations is given in the third column of Table 4 . The second column of Table 4 gives the integral representation solution from Eq. (90), which is also obtained from the invariant difference equation (105). The fourth column of Table 4 gives the results computed with the Rayleigh-Ritz method, that is, from Eq. (107). The results given in Table 4 computed with the standard difference equation (117) required solving a system of seven algebraic equations. The Rayleigh-Ritz method of Eq. (107) required solving two algebraic equations, but the results are sensitive to the form chosen for the trial function. Because the invariant difference equation (105) holds exactly for any arbitrary grid spacing, the results at the grid points can be computed

\begin{tabular}{|c|c|c|c|}
\hline \multicolumn{4}{|c|}{ TABLE 4. Comparison of Numerical Solutions of $\left(x^{4}+1\right) y^{\prime \prime}+4 x^{3} y^{\prime}+e^{-x}=0$. } \\
\hline$x$ & $\begin{array}{c}y(x) \text { With Invariant } \\
\text { Difference } \\
\text { Equation (105) }\end{array}$ & $\begin{array}{c}y(x) \text { With Standard } \\
\text { Difference } \\
\text { Equation (117) }\end{array}$ & $\begin{array}{c}y(x) \text { With Rayleigh- } \\
\text { Ritz Variational } \\
\text { Method (107) }\end{array}$ \\
\hline 0 & 0 & 0 & 0 \\
\hline $1 / 8$ & 0.035027 & 0.035117 & 0.035877 \\
\hline $1 / 4$ & 0.056220 & 0.056420 & 0.056004 \\
\hline $3 / 8$ & 0.065180 & 0.065484 & 0.063685 \\
\hline $1 / 2$ & 0.063560 & 0.063920 & 0.061743 \\
\hline $5 / 8$ & 0.053446 & 0.053781 & 0.052567 \\
\hline $3 / 4$ & 0.037537 & 0.037771 & 0.038160 \\
\hline $7 / 8$ & 0.018841 & 0.018944 & 0.020184 \\
\hline 1 & 0 & 0 & 0 \\
\hline
\end{tabular}
sequentially, not simultaneously, without an algebraic system with Eq. (105). 


\section{CONCLUSION}

The concept of differential-difference invariants allows one to incorporate invariance properties of second-order differential equations into discretized simulations of two-point boundary value problems. Group invariant discritizations have been shown to yield exact results for two-point eignvalue problems and superconvergent numerical results for two-point inhomogeneous boundary value problems. 
This report has been reproduced directly from the best available copy. It is available electronically on the Web (http://www.doe.gov/bridge).

Copies are available for sale to U.S. Department

of Energy employees and contractors from:

Office of Scientific and Technical Information

P.O. Box 62

Oak Ridge, TN 37831

(865) 576-8401

Copies are available for sale to the public from: National Technical Information Service

U.S. Department of Commerce

5285 Port Royal Road

Springfield, VA 22161

(800) 553-6847 


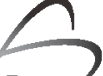 \\ - Los Alamos NATIONAL LABORATORY}

